\title{
Improvement of Fading Channel Modeling Performance for Wireless Channel
}

\author{
Inaam Abbas Hieder \\ Department of Computer Science, College of Medicine, University of Baghdad, Iraq
}

\begin{tabular}{l}
\hline \hline Article Info \\
\hline Article history: \\
Received Dec 13, 2017 \\
Revised Feb 24, 2018 \\
Accepted Mar 15, 2018 \\
\hline
\end{tabular}

\section{Keyword:}

Communication channels Fading

Frequency-selective fading channels

Rayleigh channels

\begin{abstract}
Fading channel modeling is generally defined as the variation of the attenuation of a signal with various variables. Time, geographical position, and radio frequency which is included. Fading is often modeled as a random process. Thus, a fading channel is a communication channel that experiences fading. In this paper, the proposed system presents a new design and simulate a wireless channel using Rayleigh channels. Rayleigh channels using two approaches (flat and frequency-selective fading channels) in order to calculate some path space loss efforts and analysis the performance of different wireless fading channel modeling. The results show that the bite error rate (BER) performance is dramatically improved in the value of signal to noise ratio (SNR) is equal to $45 \mathrm{~dB}$. Finally, the experimental results show that the proposed method enhances the performance of fading channel modeling by reducing the error of BER when the SNR is reduced also. Moreover, the more accurate model is Rayleigh model which can be considered for developing fading channel model.
\end{abstract}

Copyright $(2018$ Institute of Advanced Engineering and Science. All rights reserved.

\section{Corresponding Author:}

Inaam Abbas Hieder,

Department of Computer Science, College of Medicine,

University of Baghdad,

Baghdad, Iraq.

Email: Inaam62@gmail.com

\section{INTRODUCTION}

Many services of the wireless industry have provided for the users by developing and deploying an infrastructure. The technological infrastructure such as design, production and deployment may more expensive thereby manufacturers looking for different alternatives in order to avoid the high costs. A simulation of a real wireless system is one of these alternatives. The pros of simulation which is allowed to less expensive testing of designs. This paper has simulated and tested fading channel modeling for wireless communication.

The Rayleigh fading is considered a good approximation of realistic channel conditions because a receiver is in motion relative to a transmitter with no line-of-sight path between their antennas for the wireless transmission system [1]. This paper first addresses the problem of enhancing the performance of fading channel modeling by reducing the error BER as well as reducing the error of SNR for fading channel simulation model. These errors are reduced by used Rayleigh fading channel. The use of it by using two different approaches (flat and selecting fading) to loss the efforts and analysis the performance of different wireless fading channel modeling.

\section{RELATED WORK}

The term Rayleigh fading channel indicates to a multiplicative distortion $\mathrm{h}(\mathrm{t})$ of the transmitted signal $s(t)$, as in $y(t)=h(t) \cdot s(t)+n(t)$, where $y(t)$ is the received waveform and $n(t)$ is the noise [2]. The effect 
of three different channel models, AWGN channel, flat fading channel, and frequency selective fading channel by using the data and image under two different scenarios was tested by [3].

So far, the most commonly used the statistical properties of Clarke's fading model with a finite number of sinusoids and an enhanced reference model is introduced for the simulation of Rayleigh fading channels was analyzed by [4]. Similar studies have been conducted by [5], a new sum-of-sinusoids statistical simulation models are introduced for Rayleigh fading channels. A compact sum-of-sinusoid (SOS) simulation model for Rayleigh and radiant fading channels was proposed by [6].

The propagation takes place in areas which are sparsely populated with scatters. They studied the statistical properties of the capacity of multipath fading channels modelled by SOC processes was introduced by [7]. A novel statistical model for the overall signal strength of the GSM uplink band depend on CF, which characterizes both wireless fading channels and MS dynamics was proposed by [8].

\section{PROPOSED SIMULATION APPROACH}

The proposed simulation approach can be divided into two main stages such as: channel and mobile channel. Rayleigh fading channel can be divided into AWGN and space loss while mobile channel can be divided into flat fading and selective fading as shown in Figure 1.

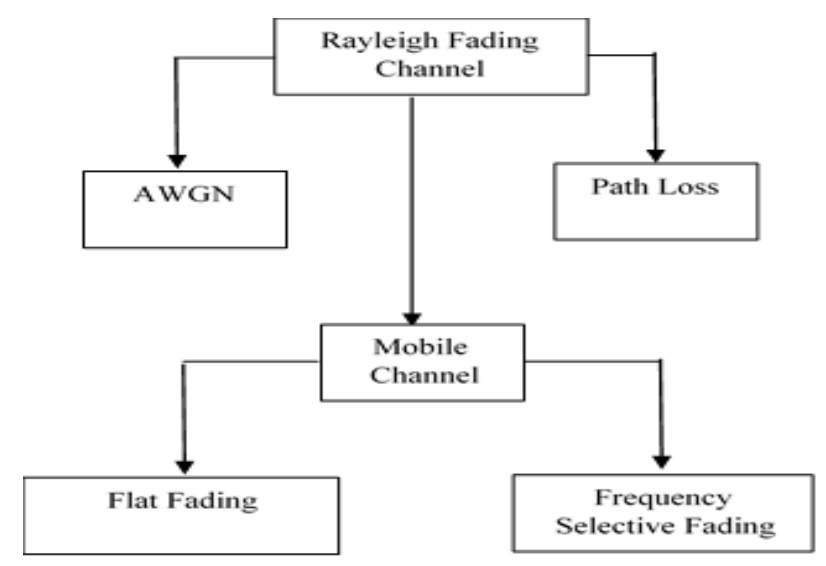

Figure 1. The stages of proposed simulation approach

\subsection{Rayleigh fading channel}

Rayleigh fading channel reflects the fading is caused by multipath reception. Thereby, Rayleigh fading channel undertakes that the magnitude of a signal which has been passed through transmission medium will differ randomly, or fade, according to a Rayleigh distribution. As well as, Rayleigh fading defined as a reasonable model when there are many objects in the environment which has scattered the radio signal before it arrives at the receiver. When there is no dominant line-of-sight propagation between the transmitter and receiver, Rayleigh fading channel is most applicable.

\subsubsection{AWGN channel}

The AWGN Channel block presents white Gaussian noise to a real or complex input signal. When the input signal is real, this block presents real Gaussian noise and generates a real output signal. While the input signal is complex, this block presents complex Gaussian noise and generates a complex output signal. This block receives its sample time from the input signal. This block accepts a scalar-valued, vector, or matrix input signal with a data kind of kind single or double. The output signal receives port data types from the signals that drive the block.

In this study, the real and imaginary parts of the complex value are assumed to be white Gaussian noise (mean is equal to zero and the same variance). The signal to noise ratio (SNR) can be computed by [9]:

$$
S N R=10 \log _{10}\left(\frac{2 E_{b}}{N_{0}}\right)
$$


Where

$\mathrm{Eb}$ : is energy data

2/NO: is the two-sided power spectral density.

The bit rate (BER) can be calculated by using the following equation:

\subsubsection{Path loss}

$$
B E R=\frac{\text { No. of Erroneous }}{\text { No. of Check Bit }}
$$

A principally significant element in the design of wireless system or a radio communication system considers as the radio signal path loss. The radio signal path loss will specify few elements of the communication such as the antennas height, the transmitter power and principally the gain. Furthermore, path loss will also affect the form of transmission utilized, the essential receiver sensitivity and some other factors. The signal path loss can be specified arithmetically and these computations are often assumed whereas designing system activities. These based on information of the signal propagation attributes. The signal radio path loss includes generally in a reduction in power density of a signal as it transmits via the environment that it is travelling. The chief reasons are the following:

a. Free space path loss: the free space path loss appears as the signal travels via space without any other effects reducing the signal. This can be supposed of as the signal growth as an ever increasing sphere. The energy in any specified area will decrease as the area enclosed becomes larger.

b. Absorption losses: when a radio signal push through a medium that it is not obvious to radio signals absorption losses appear.

c. Diffraction: These losses appear when an object occurs in the signal's path. The signal can diffract nearby the object, but losses appear. The loss is greater the more rounded the object. Concerning sharp edges, the radio signals are likely to diffract better.

d. Multipath: signals will reach the receiver through several variant paths because a lot of reflections. These signal reflections can add or subtract from each other based on the relative phases of the signals. The clear example is cellular telecommunication phones, due to they are subjected to this effect that is recognized as Rayleigh fading.

e. Atmosphere: the atmosphere was affected in radio signal paths. Below $30-50 \mathrm{MHz}$, lower frequencies, and the ionosphere has an important effect, refracting them return back to the earth. Above $50 \mathrm{MHz}$, the troposphere has a main effect, refracting also the signals return back to the earth.

f. Obstacles: buildings and vegetation have an amazing effect. The buildings will absorb and not only reflect the signal also. In context, principally when trees, we, and foliage may reduce radio signals. Furthermore, the hills, clearly will check the path and significantly reduce the signal, perhaps creating the reception impossible.

\subsection{Mobile channel}

Mobile channels operate via electromagnetic radiation from the transmitter to the receiver. In practical, one might solve the electromagnetic field equations, in combination with the transmitted signal, to get the electromagnetic field imposing on the receiver antenna. This would have to be completed taking into account the obstructions produced by ground, buildings, vehicles, etc. in the vicinity of this electromagnetic wave.

\subsubsection{Flat fading}

Flat fading can be defined as the case when the channel coherence bandwidth is greater than the signal bandwidth and later all frequencies of the transmitted signal knowledge the same channel condition that is over the signal bandwidth, the channel frequency response is basically flat; and therefore the name Flat Fading. Moreover, this matches to having an expected smaller than the signal symbol period. The flat fading can be computed by [10] as follows:

$$
y(t)=\int_{-\infty}^{\infty} X(f) H(f, t) e^{j 2 \pi f t} d f
$$

where $X(f)$ can be defined as the Fourier transform of $x(t)$ and $H(f, \tau)$ can be defined as the Fourier transform of $h(t, \tau)$. Since a flat channel verifies the condition that $\mathrm{W}<<\mathrm{f}$ coh, all the frequency components of undertake basically the same reduction and phase shift during transmission. Thus, it infers that within the 
bandwidth performed by $X(f)$, the time different channel transfer function $\mathrm{H}\left(\mathrm{f}_{\mathrm{t}}\right)$ is constant in the frequency variable. Hence

$$
\begin{aligned}
& H(f, t)=H(0, t)=H(t) \\
& y(t)=H(t) \int_{-\infty}^{\infty} x(f) e^{j 2 \pi f t} d f \\
& y(t)=H(t) x(t)
\end{aligned}
$$

As mentioned above, $H(t)$ is complex - valued. Thereby, $H(t)=\alpha(t) e^{j \phi(t)}$.

$$
y(t)=\alpha(t) e^{j \phi(t)} x(t)
$$

This specifies a multiplicative channel model. By using additive Gaussian noise, the flat fading channel model is displayed in Figure 2. If the channel is gradually variable, the amplitude reduction and phase shift is basically constant during one symbol interval and coherent detection can be applied. The channel model is given by [11], [12] as follows:

$$
y_{k}=\alpha_{k} x_{k}+n_{k} \quad \mathrm{k}=1,2 \ldots \mathrm{N}
$$

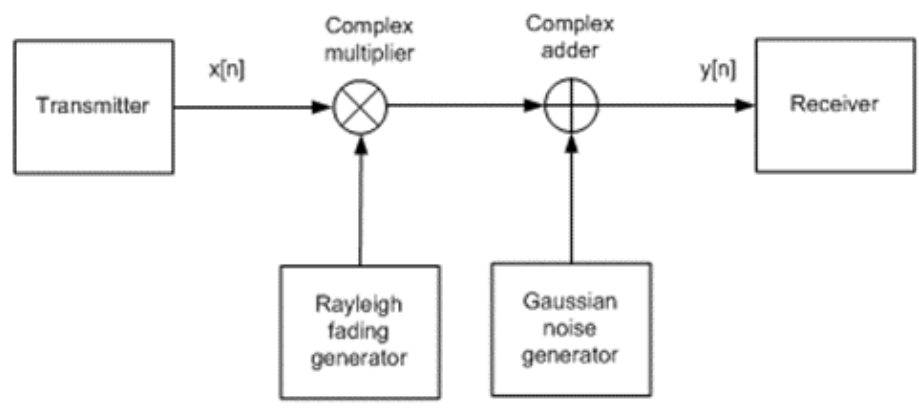

Figure 2. Flat fading channel

\subsubsection{Frequency-selective fading channels}

For radio transmission, the channel spectral reply is not flat. This channel has dips or fades in the reply because the reflections producing cancellation of positive frequencies at the receiver. These reflections off near-by objects such as ground, buildings, trees, etc. is produced to multipath signals of similar signal power in which is called the direct signal. Moreover, this may result in deep nulls in the received signal power because negative interference. On behalf of narrow bandwidth transmissions if the null in the frequency reply appears at the transmission frequency then the total signal may be lost. This problem may be fairly overcome in two ways.

Through transmitting a wide bandwidth signal or spread spectrum such as CDMA, any dips in the spectrum only produce in a slight loss of signal power, rather than that a whole loss. Another different method is to divide the transmission up into many slight bandwidth carriers, as is completed in a COFDM/OFDM transmission. The original signal is put on a wide bandwidth thereby, any nulls in the spectrum are candidate to appear at all of the carrier frequencies. This case will result in only specific of the carriers being lost, rather than that the total signal. The information in the lost carriers can be improved produced enough forward error corrections is sent.

On behalf of the transmitted signal, has bandwidth $\mathrm{W}$ bigger than the coherence bandwidth fcoh of the channel, the frequency mechanisms of, with frequency separation reaching to fcoh are exposed to variant gains and phase shift. In this case, the channel is called to be frequency selective. Addition distortion is produced by the time difference that is the fading effect on the evidenced such as a time variation in the 
received power signal of the frequency mechanisms in. When $\mathrm{W}>>$ fcoh, the multipath mechanisms in the channel reply are divided in delay by at least $1 / \mathrm{W}$. In this case, the selection theorem can be utilized to represent the resolvable received signal mechanisms. Such a development produced to an illustration of the time-varying channel impulse.

$$
h(t ; \tau)=\sum_{i=1}^{L} c_{i}(t) \delta(\tau-i / w)
$$

And the matching time variant transfer function is given by Equation (10):

$$
h(t ; \tau)=\sum_{i=1}^{L} c_{i}(t) e^{j 2 w t f i / w}
$$

where ci can be defined as the complex valued channel gain of the $\mathrm{i}$ th multipath element and $\mathrm{m}$ can be defined as the number of resolvable multipath elements. Meanwhile the multipath spread Tm and the time resolution of the multipath can be defined as 1/W, as shown in Equation (11) as bellows:

$$
m=\left[T_{m} W\right]+1
$$

A channel having the impulse reply is given by Equation (9) can be represented by a tapped-delay line with $\mathrm{L}$ taps and complex valued, time-variant tap coefficients ci(t). Figure 3 shows the tapped-delayline channel model that is suitable for the frequency selective fading channel. The randomly time variant tap gains ci(t) can also be represented by Equation (12) as bellows:

$$
c_{i}(t)=\alpha(t) e^{j \phi i(t)} \mathrm{i}=1,2 \ldots, \mathrm{m}
$$

where $c_{i}(t)$ denote the amplitudes and $\varphi_{i}(t)$ denote the phase matching phases. For the frequency selective fading channel, each of the tap gain has a magnitude that is showed as Rayleigh fading [13].

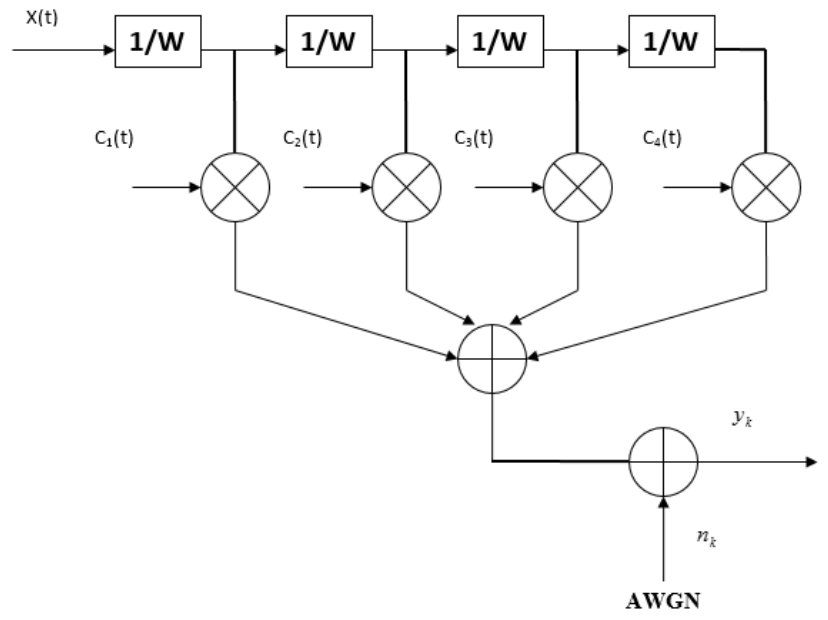

Figure 3. Tapped-delay-line channel model

\section{EXPERMENTAL RESUTLS}

The performance evaluation of channel is displayed in Figure 4. The AWGN, path loss, and mobile channel can be appeared. 


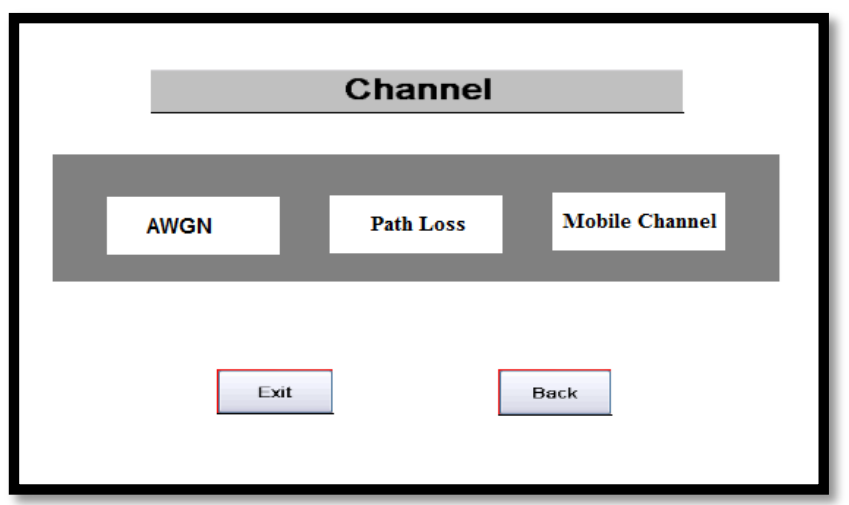

Figure 4. The simulation channel of user interface

\subsection{Performance evaluation with AWGN}

Figure 5 shows the physical part of the random additive white Gaussian noise (AWGN) for the channel produced that was shown the mean $(\mathrm{m})$ is equal to zero and the variance $(\sigma 2)$ is equal to one. While Figure 6 displays the normalized probability density function of AWGN that is added to receive baseband signal.
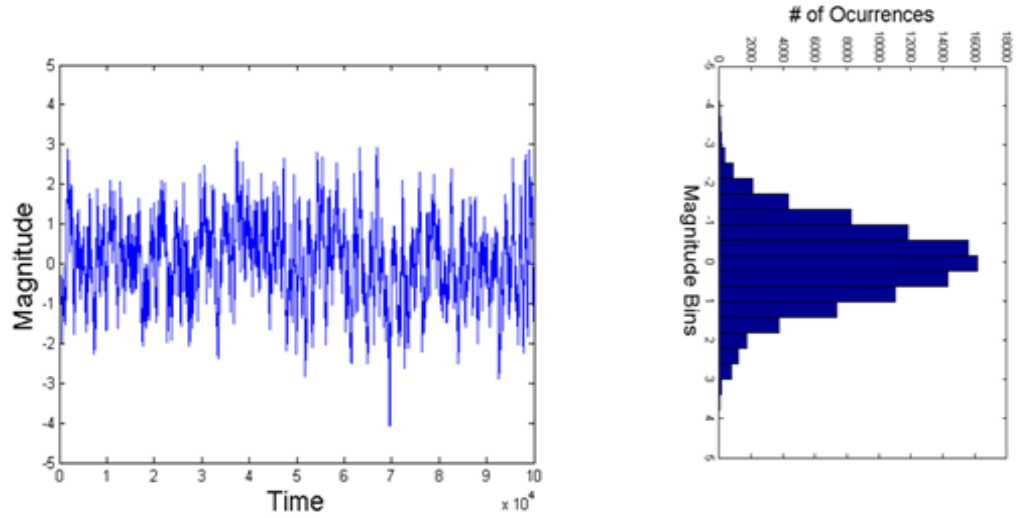

Figure 5. The random additive white Gaussian noise with mean $(\mathrm{m}=0)$ and variance $(\sigma 2=1)$

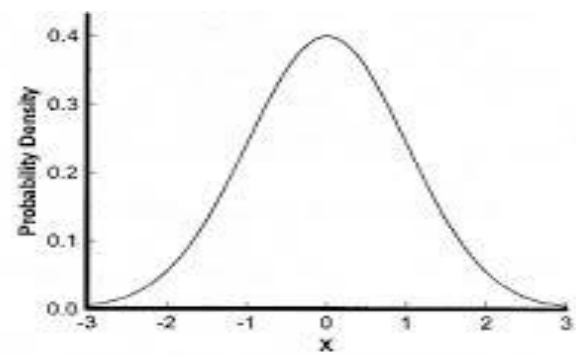

Figure 6 . The standard normal probability density function $(\sigma 2=1)$

A further method to show that the frequency reply remains white after multiplying the time area function with a rectangular window is to detect the autocorrelation function in each case: a uniform function can be defined as the autocorrelation function for an AWGN signal is an impulse as well as the frequency reply of an impulse. Adding zeros to the AWGN function does not replace the result from being an impulse, and therefore the frequency reply will still be constant (white). Adding zeros does introduce between the 
existing samples in frequency as well as the paths previously called are generated and to give the window size of length $T$ of an AWGN signal and the samples in frequency divided by $1 / T$ will still independent, but all samples will be displayed as dependent on between the two adjacent samples divided by $1 / \mathrm{T}$.

As shown in Figure 7 the performances evaluation of the AWGN channel with mean $(\mathrm{m}=0)$ and variance $(\sigma 2=1)$. The performance evaluation of this channel is applied by transmitting 100000 bits. It can be displayed from the figure; the value of SNR is $12 \mathrm{~dB}$ for bit error rate (BER) between $\left(10^{-5}\right)$.

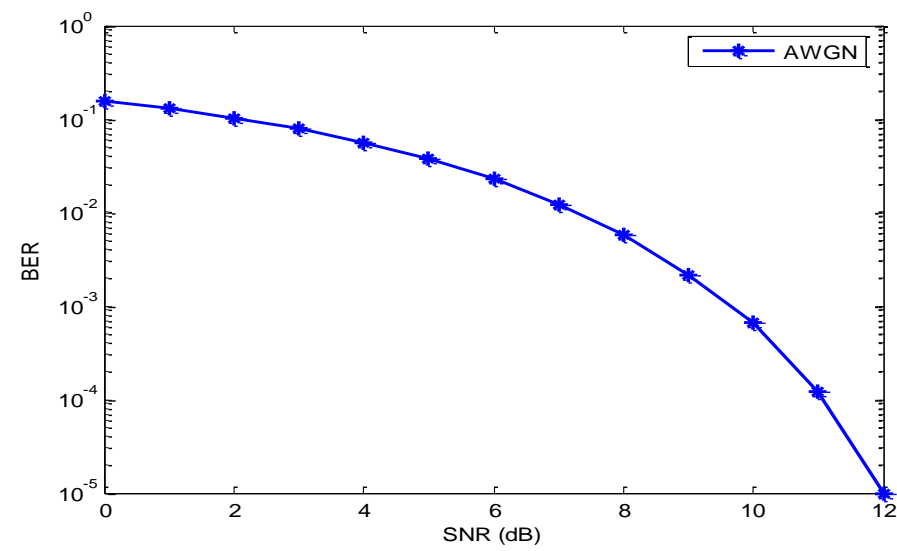

Figure 7. Performance evaluation with AWGN $\left(\mathrm{SNR}=12 \mathrm{~dB}\right.$ and $\mathrm{BER}=\left(0-10^{-5}\right)$

\subsection{Performance evaluation of path loss} as follows:

The performance evaluation of path loss has been calculated by using Equation (13) as given by [13]

$$
P_{r}(d)=\frac{P_{t} G_{r} G_{t} \lambda^{2}}{(4 \pi d)^{2} L}
$$

It is drawn with the distance in $(\mathrm{Km})$ as shown in Figure 8. This figure shows that the distance increases with the receiver power $\mathrm{dB}$ is increased also. Moreover, this figure considers variant values of transmitter power $(\mathrm{Pt}=3,5,7 \mathrm{~W})$ and the root station antenna height $(100 \mathrm{~m})$.

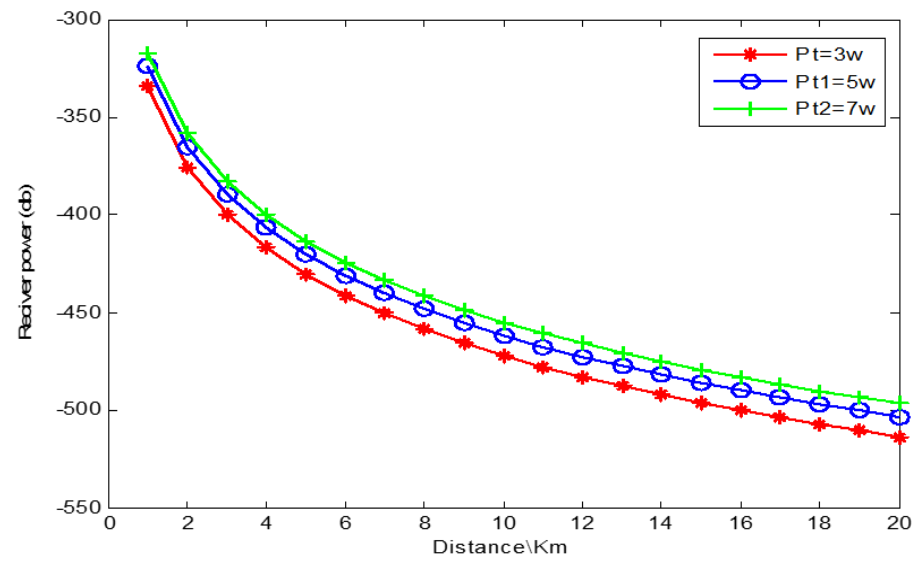

Figure 8 . The relation between the receiver powers in $\mathrm{dB}$ depends on the $\mathrm{Pt}$ and distance $(\mathrm{Km})$

Figure 9 displays the mobile station antenna with height $(\mathrm{Hm}=1$ to $10 \mathrm{~m})$ and power transmitted with $(5 \mathrm{~W})$. It is clear from this figure, the effect of the mobile station antenna height $(\mathrm{Hm})$ with respect to receiver power.

Improvement of Fading Channel Modeling Performance for Wireless Channel (Inaam Abbas Hieder) 


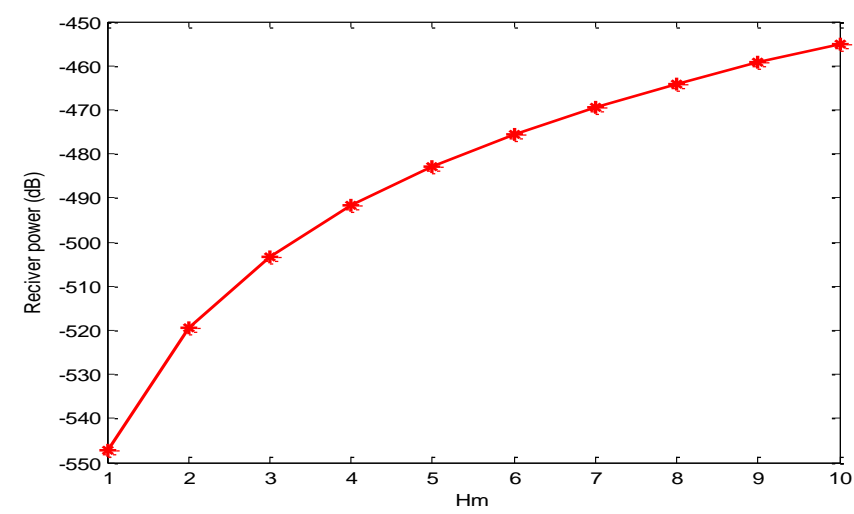

Figure 9. The relation between the receiver power in $\mathrm{dB}$ and the height $\mathrm{Hm}$

\subsection{Performance evaluation with mobile channel}

Figure 10 displays the performance evaluation of flat fading with BPSK modulation. The performance evaluation of this system is applied by transmitting 100000 data bits. It is clear from this figure; the value of SNR is equal to $45 \mathrm{~dB}$ at $\mathrm{BER}=10^{-5}$.

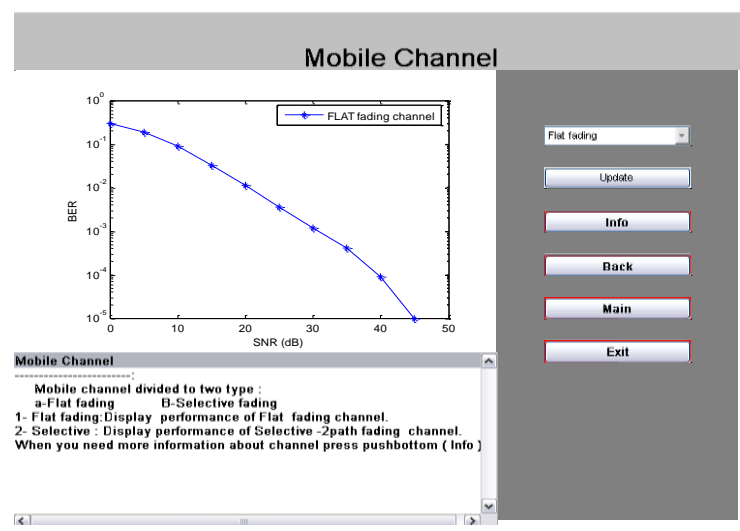

Figure 10. Performance evaluation of flat fading channel

Figure 11 displays the performance evaluation of frequency selective fading with BPSK modulation. This system is applied by transmitting 10000 data bits. Thus, it can be shown from this figure, the value of SNR is equal to $49 \mathrm{~dB}$ for $\mathrm{BER}=10^{-4}$.

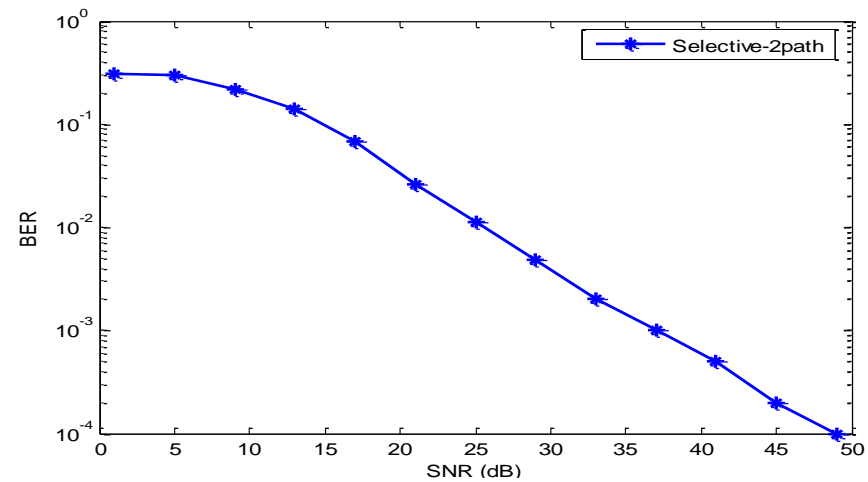

Figure 11. Performance evaluation of frequency selective fading channel 


\section{CONCLUSION}

The current work uses AWGN, path loss, flat fading channel and frequency selective fading to simulate a wireless channel. From the results, it could be seen that AWGN and path loss have a huge effect on performances of simulation wireless channel. Moreover, flat fading and frequency selective fading to reduce the error bit noise and the BER which was successfully met by the given methods. The experimental results show that AWGN, path loss, flat fading channel and frequency selective fading are good methods to simulate a wireless channel with respect to the performance evaluation for each method (SNR and BER).

\section{REFERENCES}

[1] D. Tse and P. Viswanath, "Fundamentals of wireless communication," Cambridge, 2004.

[2] M. Patzold, "Mobile fading channels," John Wiley \& Sons, Inc, 2003.

[3] M. S. Miah, et al., "Performance comparison of AWGN, flat fading and frequency selective fading channel for wireless communication system using 4QPSK," International Journal of Computer and Information Technology, vol. 1, no. 2, pp. 82-90, 2011.

[4] C. Xiao, et al., "Novel sum-of-sinusoids simulation models for Rayleigh and Rician fading channels," IEEE Transactions on Wireless Communications, vol. 5, no. 12, 2006.

[5] C. Xiao, et al., "Statistical simulation models for Rayleigh and Rician fading," International Conference on the Communications, ICC'03. IEEE, 2003.

[6] A. Alimohammad, et al., "An accurate and compact Rayleigh and Rician fading channel simulator," Conference on the Vehicular Technology, VTC Spring 2008. IEEE, 2008.

[7] R. Hicheri, et al., "On the statistical properties of the capacity of sparse multipath fading channels," International Conference on the Advanced Technologies for Communications (ATC), 2014.

[8] S. Yin, et al., "Statistical modeling for spectrum usage characterizing wireless fading channels and mobile service dynamics," IEEE Transactions on Vehicular Technology, vol. 62, no. 8, pp. 3800-3812, 2013.

[9] L. W. Couch, et al., "Digital and analog communication systems, vol. 6, 1997.

[10] Sklar, B. Rayleigh fading channels in mobile digital communication systems," I. Characterization. IEEE Communications magazine, vol. 35, no. 9, pp. 136-146, 1997.

[11] T. S. Rappaport, "Wireless Communications--Principles and Practice, (The Book End)," Microwave Journal, vol. 45 , no. 12 , pp. 128-129, 2002

[12] S. Gaur, "Analysis of Advanced Diversity Receivers for Fading Channels," 2003.

[13] J. Yun, "Adaptive Resource Allocation for D-TDD Systems in Wireless Multimedia Networks,” 2004. 